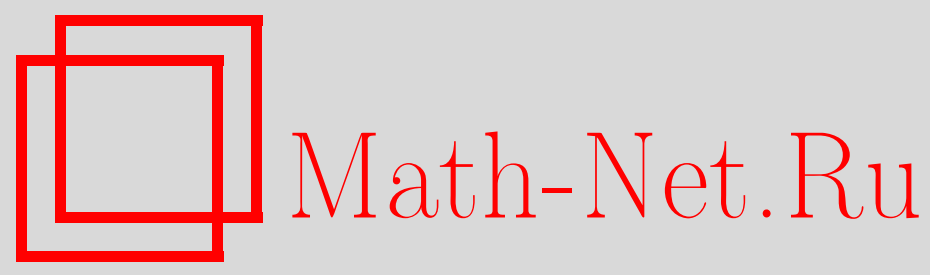

О. Г. Парфенов, Оценки спектра некоторых дифференциальных операторов, Матем. заметки, 1997, том 61, выпуск 4, 634-636

DOI: https://doi.org/10.4213/mzm1543

Использование Общероссийского математического портала Math-Net.Ru подразумевает, что вы прочитали и согласны с пользовательским соглашением http://www . mathnet.ru/rus/agreement

Параметры загрузки:

IP: 54.84 .234 .179

26 апреля 2023 г., 16:59:14

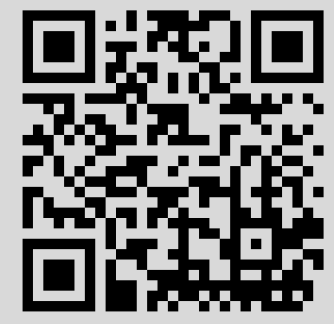




\section{ОЦЕНКИ СПЕКТРА НЕКОТОРЫХ ДИФФЕРЕНЦИАЛЬНЫХ ОПЕРАТОРОВ}

\section{О.Г. Парфёнов}

Рассмотрим задачу о спектре дифференциального уравнения вида

$$
-\left(\left(1-x^{2}\right)^{\alpha} u^{(l)}\right)^{(l)}+p(x) u=\lambda u .
$$

При условиях, которые мы укажем ниже, спектр (1) является дискретньм. Возникает задача дальнейшего исследования свойств спектра (1). Например, можно исследовать необходимые и достаточные условия, при которых сходятся ряды

$$
\sum_{n}\left|\lambda_{n}\right|^{-\rho}, \quad \rho>0, \quad \sum_{n}\left|\lambda_{n}\right|^{-\rho_{n} \tau / \rho-1}, \quad \rho>0, \quad \tau>0 .
$$

Вариационная постановка этой задачи (более удобная для применения методов функционального анализа) такова: исследовать поведение сингулярньх чисел ( $s$-чисел) оператора вложения $J: \stackrel{\circ}{W}_{2, \alpha}^{l}(-1,1) \rightarrow L_{2}(\mu), d \mu=p(x) d x$. Класс $\stackrel{\circ}{W}{ }_{2, \alpha}^{l}(-1,1)$ есть замыкание множества $C_{0}^{\infty}(-1,1)$ по норме

$$
\|u\|_{W_{2, \alpha}^{l}}=\left(\int_{-1}^{1}\left(\left|u^{(l)}\right|^{2}\left(1-x^{2}\right)^{-\alpha}+|u|^{2}\left(1-x^{2}\right)^{-2 l-\alpha}\right) d x\right)^{1 / 2} .
$$

Рассмотрим сразу многомерньй вариант этой задачи (ему также отвечает некоторьй дифференциальный оператор в частньх производных).

Пусть $\Omega$ - открытое множество в $\mathbb{R}^{m}$. Обозначим $d_{x}$ - расстояние от точки $x$ до гранищы области $\Omega$

$$
d_{x}=\inf _{y \in \partial \Omega}|x-y|
$$

если $\Omega=\mathbb{R}^{m}$, положим $d_{x}=1+|x|$. Определим класс $\stackrel{\circ}{W_{p}^{l}}{ }_{p, \lambda}(\Omega)$, следуя работе В. Л. Олейника и Б. С. Павлова $[1]$, как замыкание $C_{0}^{\infty}(\Omega)$ по норме

$$
\|f\|_{W_{p, \lambda}^{l}(\Omega)}=\left(\int_{\Omega}\left(\sum_{|\beta|=l}\left|D^{\beta} f\right|^{p} d_{x}^{-\lambda}+|f|^{p} d_{x}^{-p l-\lambda}\right) d x\right)^{1 / p}
$$

здесь $l=1,2,1 \leqslant p<\infty, \lambda \in(-\infty, \infty)$. При $p=\infty$ обычная модификация. Определим также классы $\stackrel{\circ}{L}_{p, \lambda}^{l}(\Omega)$ как замыкание $C_{0}^{\infty}(\Omega)$ по норме

$$
\|f\|_{L_{p, \lambda}^{l}(\Omega)}=\left(\int_{\Omega}\left(\sum_{|\beta|=l}\left|D^{\beta} f\right|^{p} d_{x}^{-\lambda}\right) d x\right)^{1 / p} .
$$

Работа выполнена при поддержке Российского фонда фундаментальных исследований. 
При $\lambda \geqslant 0$ нормы (2), (3) эквивалентны (см., [1]). При $\lambda=0$, таким образом, мы рассматриваем обычный класс Соболева $\stackrel{\circ}{W}_{p}^{l}(\Omega)$.

Для классов $\stackrel{\circ}{W}_{p, \lambda}^{l}(\Omega)$ в работе [1] получена теорема вложения в пространство $L_{q}(\Omega, \mu)$ при условии $p l>m$. Подчеркнем, что $\mu$ - произвольная борелевская мера в $\Omega$. Для формулировки теоремы вложения рассмотрим конечнократное покрытие области $\Omega$ шарами

$$
\Omega=\bigcup_{n} B_{n}: \quad B_{n}=\left\{y|| x_{n}-y \mid<r_{n}\right\}
$$

причем $r_{n}=(1 / 4) d_{x_{n}}$. Такое покрытие называется покрытием типа Уитни. (Вместо $1 / 4$ можно взять любое число $\gamma, 0<\gamma<1$.) Далее, определим последовательность

$$
\beta_{n}=r_{n}^{(p l+\lambda-m) / p}\left(\mu\left(B_{n}\right)\right)^{1 / q} .
$$

Teоpema 1 [1]. Пycmb $p l>m$. Тогдa

а) если $1 \leqslant q \leqslant p$, то оператор вложсения $J$ класса $\stackrel{\circ}{W}_{p, \lambda}^{l}(\Omega)$ в $L_{q}(\Omega, \mu)$ ограничен в том и только том случае, если

$$
\sup _{n} \beta_{n}<\infty
$$

$\left.\mathrm{a}^{\prime}\right)$ если $1 \leqslant q \leqslant p$, то оператор вложения $J$ компактен тогда и только тогда, когда $\beta_{n} \rightarrow 0, n \rightarrow \infty$;

б) если $1 \leqslant p<q \leqslant \infty$, то оператор вложсения $J$ ограничен тогда и только mогда, когда

$$
\sum_{n} \beta_{n}^{r}<\infty, \quad r^{-1}=p^{-1}-q^{-1}
$$

$\left.\sigma^{\prime}\right)$ если $1 \leqslant p<q \leqslant \infty$, то оператор вложения $J$ компактен тогда и только тогда, когда выполнено условие (4).

ЗАмЕчАниЕ 1. По теореме вложения Соболева условие $p l>m$ есть критерий ограниченности вложения пространства $\stackrel{\circ}{W} \underset{p, \lambda}{l}(\Omega)$ в $C(\Omega)$, и, как нетрудно заметить, то же верно для классов $\stackrel{\circ}{W} \underset{p, \lambda}{l}(\Omega)$. Таким образом, в теореме 1 содержателен лишш вопрос о поведении меры $\mu$ вблизи границы $\Omega$.

ЗАмЕчАниЕ 2 . При условии $p l \leqslant m$ критерий ограниченности вложения $\stackrel{\circ}{W}_{p}^{l}(\Omega)$ в $L_{q}(\Omega, \mu)$ (для любой борелевской меры $\mu$ ) был получен В. Г. Мазьей (см. $\left.[2, \S 1.1, \S 1.2]\right)$ в терминах емкости и усовершенствован в работе Э. Сойера [3].

ЗАмЕчАниЕ 3. Напомним, что критерий компактности гарантирует дискретность спектра.

В данной работе мы рассматриваем вопрос принадлежности оператора вложения $J: W_{2, \lambda}^{l}(\Omega) \rightarrow L_{2}(\Omega, \mu)$ классу $\sigma_{\rho}, \rho>0$.

Напомним определение операторного идеала $\sigma_{\rho}$ : пусть $H_{1}, H_{2}$ - гильбертовы пространства, $T: H_{1} \rightarrow H_{2}-$ компактньй оператор,

$$
T=\sum_{n} s_{n}(T)\left(\cdot, e_{n}\right) g_{n}
$$


- его разложение Шмидта $\left(\left\{e_{n}\right\}_{1}^{\infty},\left\{g_{n}\right\}_{1}^{\infty}\right.$-ортонормированные системы в $H_{1}, H_{2}$ соответственно, $\left\{s_{n}(T)\right\}_{1}^{\infty}$ - убывающая последовательность положительных чисел, число $s_{n}(T)$ называется $n$-м сингулярным числом оператора $T$, см. подробнее об этом в $[5$, c. 161]). Говорим, что $T \in \sigma_{\rho}, \rho>0$, если $\sum_{n} s_{n}^{\rho}(T)<\infty$. Соответственно идеал $\sigma_{\rho, \tau}$, $\rho>0, \tau>0$, определяется как класс операторов $T$, для которых

$$
\sum_{n}\left(s_{n}(T)\right)^{\tau} n^{\tau / \rho-1}<\infty
$$

Наконец, идеал $\sigma_{\rho, \infty}, \rho>0$, определяется как класс операторов $T$, для которых

$$
\sup _{n} s_{n}(T) n^{-\rho}<\infty
$$

Приведем основной результат работы.

Теорема 2. Пусть $2 l>m, \rho_{*}=(l-m / 2)^{-1}$. Тогда оператор вложсения $J \in \sigma_{\rho}$, $\rho>\rho_{*}$, тогда и только тогда, когда

$$
\sum_{n} \beta_{n}^{\rho / 2}<\infty
$$

Далее, $J \in \sigma_{\rho, \tau}, \rho>\rho_{*}, \tau>0$, если

$$
\sum_{n}\left(\beta_{n}\right)^{\tau / 2} n^{\tau / \rho-1}<\infty
$$

$u T \in \sigma_{\rho_{*}, \infty}$, ecлu

$$
\sup _{n} \beta_{n} n^{-2 \rho_{*}}<\infty
$$

Доказательство теоремы 2 основано на методах работы автора [5]. Отметим лишь роль предельного показателя $\rho_{*}$. При $\rho<\rho_{*}$ уже оператор вложения, отвечаюший мере Дирака в любой точке области $\Omega$, непринадлежит $\sigma_{\rho}$, так как нет вложения в $C(\Omega)$, хотя условие (5), очевидно, выполнено.

Псковский педагогический институт

Поступило

12.09 .96

\section{СПИСОК ЦИТИРОВАННОЙ ЛИТЕРАТУРЫ}

1. Олейник В. Л., Павлов Б. С. О критериях ограниченности и полной непрерывности некоторых операторов вложения // Проблемы математической физики. № 4. Л.: Изд-во ЛГУ, 1970. С. 112-118. 2. Мазья В. Г., Шапошникова Т. О. Мульипликаторы в пространствах дифференцируемых функций. Л.: Изд-во ЛГУ, 1986. 3. Sawyer E. // Ind. Univ. Math. J. 1984. V. 33. №3. Р. 353-366. 4. Гохберг И. Ц., Крейн М. Г. Введение в теорию линейных несамосопряженньх операторов. М.: Наука, 1965. 5. Парфёнов О. Г. // Алгебра и анализ. 1991. Т. 3. № 2. C. 199-227. 